Article

\title{
Linguistic Neutrosophic Cubic Numbers and Their Multiple Attribute Decision-Making Method
}

\section{Jun Ye}

Department of Electrical and Information Engineering, Shaoxing University, 508 Huancheng West Road, Shaoxing 312000, China; yehjun@aliyun.com or yejun@usx.edu.cn; Tel.: +86-575-8832-7323

Received: 25 August 2017; Accepted: 6 September 2017; Published: 8 September 2017

\begin{abstract}
To describe both certain linguistic neutrosophic information and uncertain linguistic neutrosophic information simultaneously in the real world, this paper originally proposes the concept of a linguistic neutrosophic cubic number (LNCN), including an internal LNCN and external LNCN. In LNCN, its uncertain linguistic neutrosophic number consists of the truth, indeterminacy, and falsity uncertain linguistic variables, and its linguistic neutrosophic number consists of the truth, indeterminacy, and falsity linguistic variables to express their hybrid information. Then, we present the operational laws of LNCNs and the score, accuracy, and certain functions of LNCN for comparing/ranking LNCNs. Next, we propose a LNCN weighted arithmetic averaging (LNCNWAA) operator and a LNCN weighted geometric averaging (LNCNWGA) operator to aggregate linguistic neutrosophic cubic information and discuss their properties. Further, a multiple attribute decision-making method based on the LNCNWAA or LNCNWGA operator is developed under a linguistic neutrosophic cubic environment. Finally, an illustrative example is provided to indicate the application of the developed method.
\end{abstract}

Keywords: linguistic neutrosophic cubic number; score function; accuracy function; certain function; linguistic neutrosophic cubic number weighted arithmetic averaging (LNCNWAA) operator; linguistic neutrosophic cubic number weighted geometric averaging (LNCNWGA) operator; decision-making

\section{Introduction}

In terms of complex objective aspects of real life, human preference judgments may use linguistic expression, instead of numerical value expression, in order to be more suitable for people's thinking habits. Hence, Zadeh [1] firstly introduced the concept of a linguistic variable and applied it to fuzzy reasoning. After that, linguistic decision analysis and linguistic aggregation operators have been proposed to solve linguistic decision-making problems [2-5]. Due to the incompleteness and uncertainty of linguistic decision environments, uncertain linguistic variables and their various aggregation operators were developed and applied to uncertain linguistic decision-making problems [6-11]. As to the extension of linguistic variables, the concept of linguistic intuitionistic fuzzy numbers and their linguistic intuitionistic multicriteria group decision-making methods were introduced in the literature [12,13], and then linguistic intuitionistic multicriteria decision-making method was proposed based on the Frank Heronian mean operator [14]. Recently, the concept of a neutrosophic linguistic number, which indicates a changeable uncertain linguistic number corresponding to some specified indeterminate range, and some weighted aggregation operators of neutrosophic linguistic numbers, were presented to solve multiple attribute group decision-making problems with neutrosophic linguistic numbers [15]. Then, the concept of a linguistic neutrosophic number, which is described independently by the truth, indeterminacy, and falsity linguistic variables, and some aggregation operators of linguistic neutrosophic numbers, were proposed to solve multiple attribute group decision-making problems with linguistic neutrosophic numbers [16,17]. 
To express vagueness and uncertainty in real life, the concept of a (fuzzy) cubic set (including the internal cubic set and external cubic set) was introduced based on the hybrid information of both partial certain and partial uncertain values in [18], where the first component is an interval/uncertain value and the second component is an exact/certain value. After that, the concept of a neutrosophic cubic set (including the internal neutrosophic cubic set and external neutrosophic cubic set), where a neutrosophic cubic number (a basic element in a neutrosophic cubic set) is composed of both the interval neutrosophic number and the single-valued neutrosophic number, and the distance measure of neutrosophic cubic sets were proposed and applied to pattern recognition $[19,20]$. Then, decision-making methods with neutrosophic cubic information were put forward based on grey relational analysis [21] and cosine measures [22], respectively.

However, all the existing linguistic variables, including: uncertain linguistic variables, linguistic intuitionistic fuzzy numbers (basic elements in a linguistic intuitionistic fuzzy set), neutrosophic linguistic numbers (basic elements in a neutrosophic linguistic set), and linguistic neutrosophic numbers (basic elements in a linguistic neutrosophic set), cannot express the hybrid information of both uncertain linguistic and certain linguistic neutrosophic numbers simultaneously in linguistic decision-making environments. Furthermore, the cubic set and neutrosophic cubic set cannot also express linguistic arguments and handle linguistic decision-making problems under linguistic environments. Hence, it is necessary to extend neutrosophic cubic sets to linguistic neutrosophic arguments. For this purpose, this study presents a new concept of a linguistic neutrosophic cubic number (LNCN), where the uncertain linguistic neutrosophic number corresponding to its first part is composed of the truth, indeterminacy, and falsity uncertain linguistic variables and the linguistic neutrosophic number corresponding to its second part is composed of the truth, indeterminacy, and falsity linguistic variables. Then, we propose the operational laws of LNCNs and the score, accuracy, and certain functions of LNCN for comparing/ranking LNCNs. Further, we present a LNCN weighted arithmetic averaging (LNCNWAA) operator and a LNCN weighted geometric averaging (LNCNWGA) operator. Moreover, we develop a decision-making method based on the LNCNWAA or LNCNWGA operator and the score, accuracy, and certain functions to solve decision-making problems with the hybrid information of both certain linguistic neutrosophic numbers and uncertain linguistic neutrosophic numbers under linguistic environments.

The rest of this paper is structured as follows: Section 2 proposes the concept of LNCN (including the internal LNCN and external LNCN), the operational laws of LNCNs, and the score, accuracy, and certain functions of LNCNs to rank LNCNs. In Section 3, we propose the LNCNWAA and LNCNWGA operators to aggregate LNCNs and discuss their properties. In Section 4, a multiple attribute decision-making method is developed based on the LNCNWAA or LNCNWGA operator under a LNCN environment. In Section 5, an example illustrates the application of the proposed method. Section 6 gives conclusions and future work.

\section{Linguistic Neutrosophic Cubic Numbers (LNCNs) and Their Operational Laws}

This section proposes the concept of LNCN, which include the internal LNCN and external LNCN, and the operational laws of LNCNs.

Definition 1. Let a linguistic term set be $S=\left\{s_{j} \mid j \in[0, p]\right\}$, where $p+1$ is an odd number/cardinality. A LNCN $h$ in $S$ is constructed as $h=(u, c)$, where $u=\left\langle\left[s_{T a}, s_{T b}\right],\left[s_{I a}, s_{I b}\right],\left[s_{F a}, s_{F b}\right]\right\rangle$ is an uncertain linguistic neutrosophic number with the truth, indeterminacy, and falsity uncertain linguistic variables $\left[s_{T a}, s_{T b}\right],\left[s_{I a}, s_{I b}\right]$, and $\left[s_{F a}, s_{F b}\right]$ for $s_{T a}, s_{I a}, s_{F a} s_{T b}, s_{I b}, s_{F b} \in S$ and $T a \leq T b, I a \leq I b, F a \leq F b ; c=\left\langle s_{T}, s_{I}, s_{F}\right\rangle$ is a linguistic neutrosophic number with the truth, indeterminacy, and falsity linguistic variables $s_{T}, s_{I}$, and $s_{F}$ for $s_{T}, s_{I}$, $s_{F} \in S$.

Definition 2. Let a LNCN be $h=\left(\left\langle\left[s_{T a}, s_{T b}\right],\left[s_{I a}, s_{I b}\right],\left[s_{F a}, s_{F b}\right]\right\rangle,\left\langle s_{T}, s_{I}, s_{F}\right\rangle\right)$ for $s_{T a}, s_{I a}, s_{F a} s_{T b}, s_{I b}, s_{F b}, s_{T}$, $s_{I}, s_{F} \in S$. Then, we call 
(1) $h$ an internal $L N C N$ if $T a \leq T \leq T b, I a \leq I \leq I b, F a \leq F \leq F b$;

(2) $h$ an external $L N C N$ if $T \notin(T a, T b), I \notin(I a, I b)$, and $F \notin(F a, F b)$.

Based on the operational laws of linguistic intuitionistic fuzzy numbers and linguistic neutrosophic numbers introduced in the existing literature [12-14,16,17], we propose the following operational laws of LNCNs.

Definition 3. Let two LNCNs be $h_{1}=\left(\left\langle\left[s_{T a 1}, s_{T b 1}\right],\left[s_{I a 1}, s_{I b 1}\right],\left[s_{F a 1}, s_{F b 1}\right]\right\rangle,\left\langle s_{T 1}, s_{I 1}, s_{F 1}\right\rangle\right)$ and $h_{2}=\left(\left\langle\left[s_{T a 2}, s_{T b 2}\right],\left[s_{I a 2}, s_{I b 2}\right],\left[s_{F a 2}, s_{F b 2}\right]\right\rangle,\left\langle s_{T 2}, s_{I 2}, s_{F 2}\right\rangle\right)$. Then, their operational laws are defined as follows:

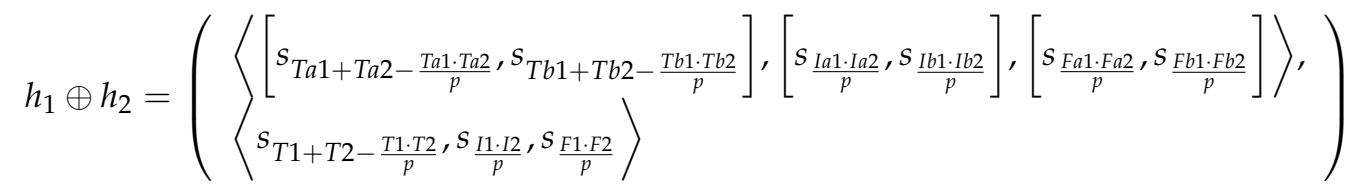

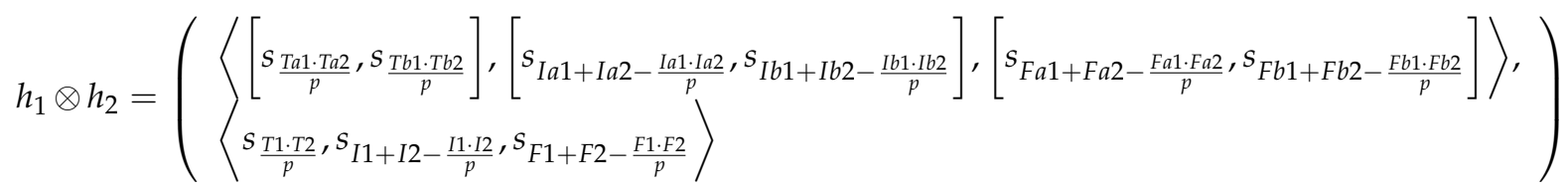

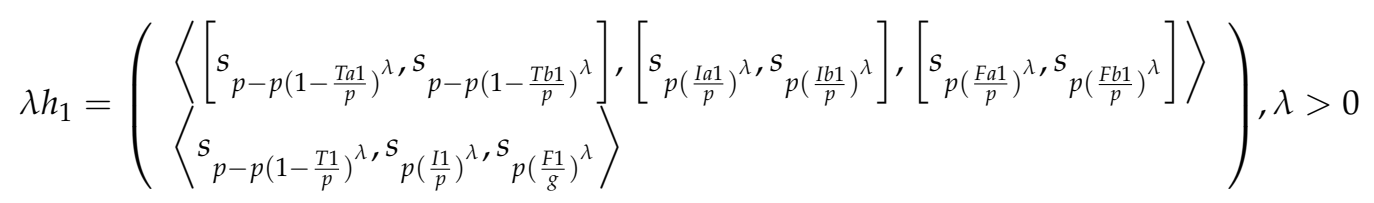

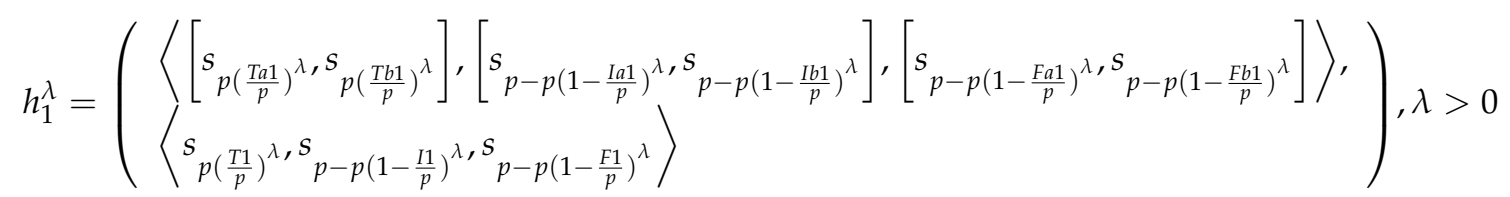

Then, the above operational results are still LNCNs.

Based on the score and accuracy functions of a linguistic neutrosophic number in the literature [16], we present the score, accuracy, and certain functions of LNCN to compare/rank LNCNs.

Definition 4. Let a LNCN be $h=\left(\left\langle\left[s_{T a}, s_{T b}\right],\left[s_{I a}, s_{I b}\right],\left[s_{F a}, s_{F b}\right]\right\rangle,\left\langle s_{T}, s_{I}, s_{F}\right\rangle\right)$ for $s_{T a}, s_{I a}, s_{F a} s_{T b}, s_{I b}, s_{F b}, s_{T}$, $s_{I}, s_{F} \in S$. Then, its score, accuracy, and certain functions are defined as follows:

$$
\begin{gathered}
S(h)=\frac{1}{9 p}[(4 p+T a+T b-I a-I b-F a-F b)+(2 p+T-I-F)], \text { for } S(h) \in[0,1] \\
H(h)=\frac{1}{3 p}[(T a+T b-F a-F b)+(T-F)], \text { for } H(h) \in[-1,1] \\
C(h)=\frac{T a+T b+T}{3 p}, \text { for } C(h) \in[0,1]
\end{gathered}
$$

Then, we introduce a ranking method based on the values of the score, accuracy, and certain functions.

Definition 5. Let two LNCNs be $h_{1}=\left(\left\langle\left[s_{T a 1}, s_{T b 1}\right],\left[s_{I a 1}, s_{I b 1}\right],\left[s_{F a 1}, s_{F b 1}\right]\right\rangle,\left\langle s_{T 1}, s_{I 1}, s_{F 1}\right\rangle\right)$ and $h_{2}=\left(\left\langle\left[s_{T a 2}, s_{T b 2}\right],\left[s_{I a 2}, s_{I b 2}\right],\left[s_{F a 2}, s_{F b 2}\right]\right\rangle,\left\langle s_{T 2}, s_{I 2}, s_{F 2}\right\rangle\right)$. Then, their ranking method based on their score, accuracy, and certain functions are defined as follows:

(1) If $S\left(h_{1}\right)>S\left(h_{2}\right)$, then $h_{1} \succ h_{2}$;

(2) If $S\left(h_{1}\right)=S\left(h_{2}\right)$ and $H\left(h_{1}\right)>H\left(h_{2}\right)$, then $h_{1} \succ h_{2}$; 
(3) If $S\left(h_{1}\right)=S\left(h_{2}\right), H\left(h_{1}\right)=H\left(h_{2}\right)$, and $C\left(h_{1}\right)>C\left(h_{2}\right)$, then $h_{1} \succ h_{2}$;

(4) $S\left(h_{1}\right)=S\left(h_{2}\right), H\left(h_{1}\right)=H\left(h_{2}\right)$, and $C\left(h_{1}\right)=C\left(h_{2}\right)$, then $h_{1} \sim h_{2}$

Example 1. Let $\left.h_{1}=\left(\left\langle\left[s_{4}, s_{6}\right],\left[s_{1}, s_{2}\right],\left[s_{1}, s_{3}\right]\right\rangle,\left\langle s_{5}, s_{1}, s_{2}\right\rangle\right), h_{2}=\left(<\left[s_{4}, s_{5}\right],\left[s_{1}, s_{2}\right],\left[s_{1}, s_{2}\right]\right\rangle,\left\langle s_{4}, s_{1}, s_{1}\right\rangle\right)$, and $\left.h_{3}=\left(<\left[s_{6}, s_{7}\right],\left[s_{2}, s_{3}\right],\left[s_{1}, s_{3}\right]>,<s_{6}, s_{2}, s_{3}\right\rangle\right)$ in the linguistic term set $S=\left\{s_{j} \mid j \in[0,8]\right\}$ are three LNCNs. Then, we need to compare them.

By using Equations (1) to (3), the values of their score, accuracy, and certain functions are as follows:

$S\left(h_{1}\right)=[32+4+6-(1+2+1+3)+16+5-(1+2)] / 72=0.7361, S\left(h_{2}\right)=[32+4+5-(1+2+1+$ $2)+16+4-(1+1)] / 72=0.7361$, and $S\left(h_{3}\right)=[32+6+7-(2+3+1+3)+16+6-(2+3)] / 72=0.7361$; $H\left(h_{1}\right)=[4+6-(1+3)+5-2] / 24=0.375, H\left(h_{2}\right)=[4+5-(1+2)+4-1] / 24=0.375$, and $H\left(h_{3}\right)=$ $[6+7-(1+3)+6-3] / 24=0.5 ;$ and $C\left(h_{1}\right)=(4+6+5) / 24=0.625$ and $C\left(h_{2}\right)=(4+5+4) / 24=0.5417$.

According to the ranking method of Definition 5, their ranking order is $h_{3} \succ h_{1} \succ h_{2}$.

\section{Two Weighted Aggregation Operators of LNCNs}

3.1. Linguistic Neutrosophic Cubic Number Weighted Arithmetic Averaging (LNCNWAA) Operator

Definition 6. Let $h_{j}=\left(\left\langle\left[s_{T a j}, s_{T b j}\right],\left[s_{I a j}, s_{I b j}\right],\left[s_{F a j}, s_{F b j}\right]\right\rangle,\left\langle s_{T j}, s_{I j}, s_{F j}\right\rangle\right)(j=1,2, \ldots, n)$ be a group of LNCNs, then the LNCNWAA operator can be defined as follows:

$$
\operatorname{LNCNWAA}\left(h_{1}, h_{2}, \cdots, h_{n}\right)=\sum_{j=1}^{n} w_{j} h_{j}
$$

where $w_{j}$ is the weight of $h_{j}(j=1,2, \ldots, n)$ for $w_{j} \in[0,1]$ and $\sum_{j=1}^{n} w_{j}=1$.

According to Definitions 3 and 6, there is the following theorem.

Theorem 1. Let $h_{j}=\left(\left\langle\left[s_{T a j}, s_{T b j}\right],\left[s_{I a j}, s_{I b j}\right],\left[s_{F a j}, s_{F b j}\right]\right\rangle,\left\langle s_{T j}, s_{I j}, s_{F j}\right\rangle\right)(j=1,2, \ldots, n)$ be a group of LNCNs, then the aggregation result obtained by Equation (4) is still a LNCN, which is calculated by the following aggregation formula:

$$
\begin{aligned}
& \operatorname{LNCNWAA}\left(h_{1}, h_{2}, \cdots, h_{n}\right)=\sum_{j=1}^{n} w_{j} h_{j}
\end{aligned}
$$

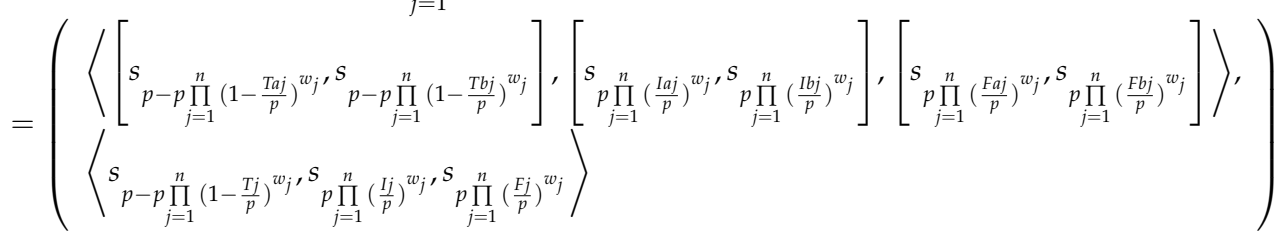

where $w_{j}$ is the weight of $h_{j}(j=1,2, \ldots, n)$ for $w_{j} \in[0,1]$ and $\sum_{j=1}^{n} w_{j}=1$.

In the following, the mathematical induction is used to prove Theorem 1.

Proof. (1) Set $n=2$, according the operational laws of LNCNs, we have the following results:

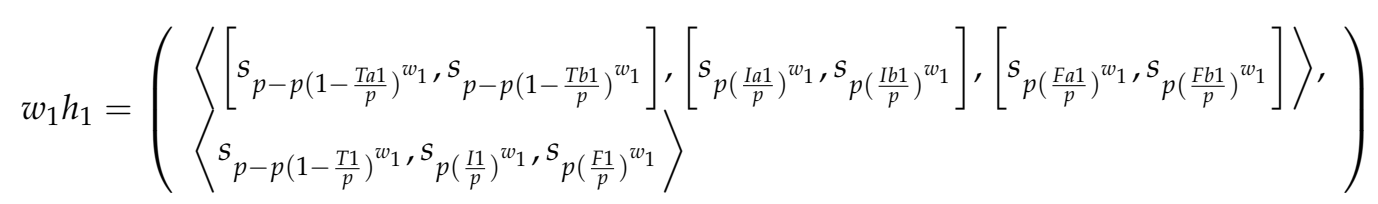




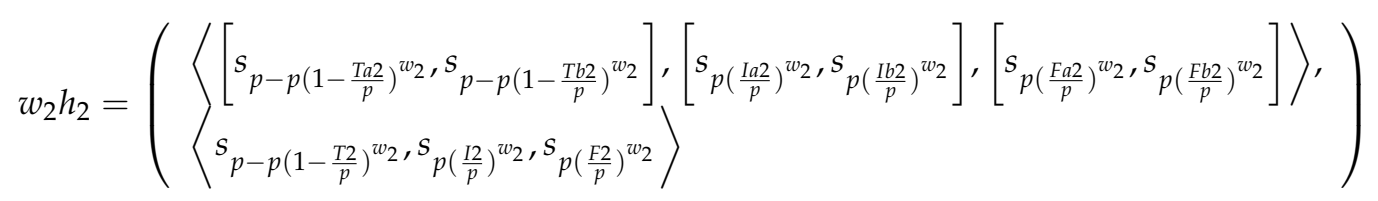

Then, there exists the following result:

$$
\begin{aligned}
& \operatorname{LNCNWAA}\left(h_{1}, h_{2}\right)=w_{1} h_{1} \oplus w_{2} h_{2}
\end{aligned}
$$

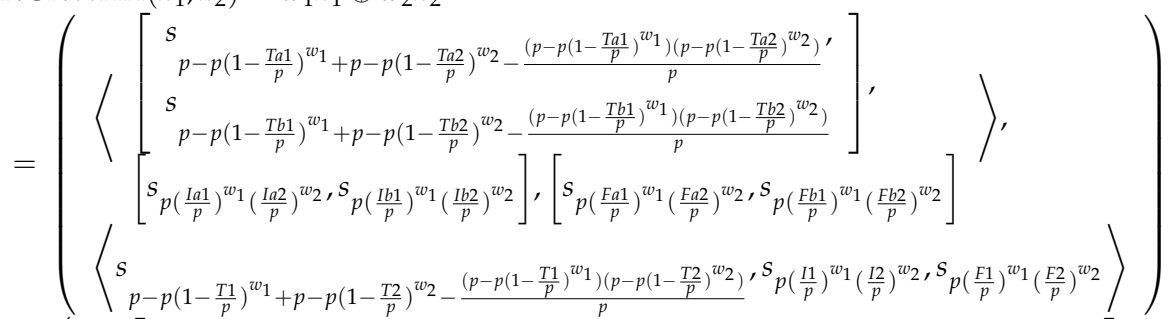

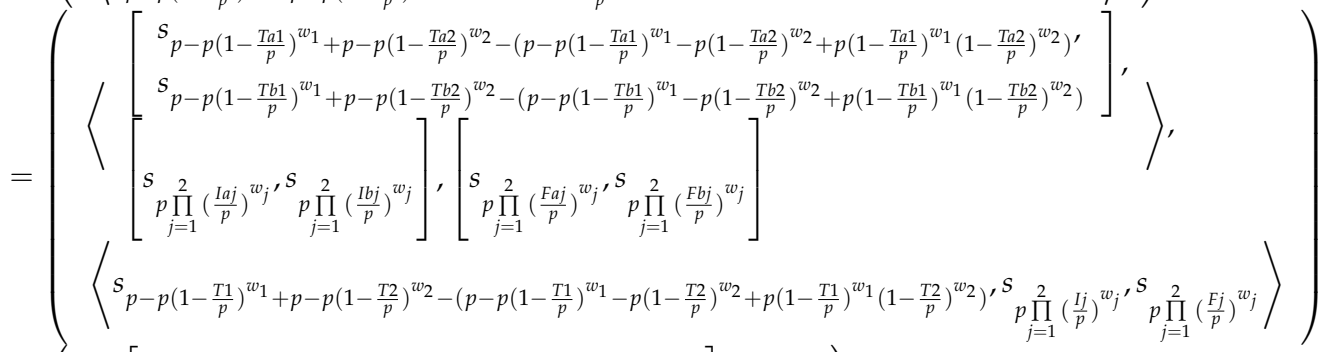

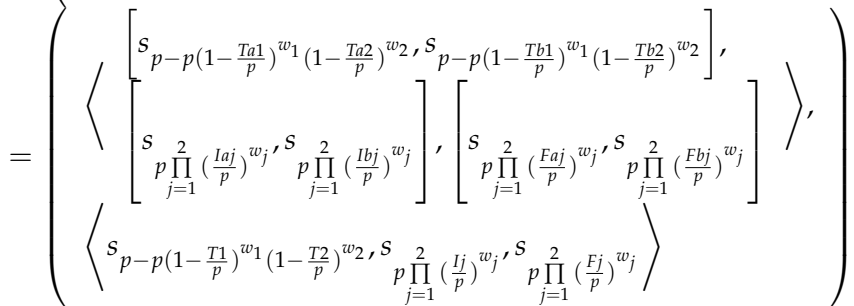

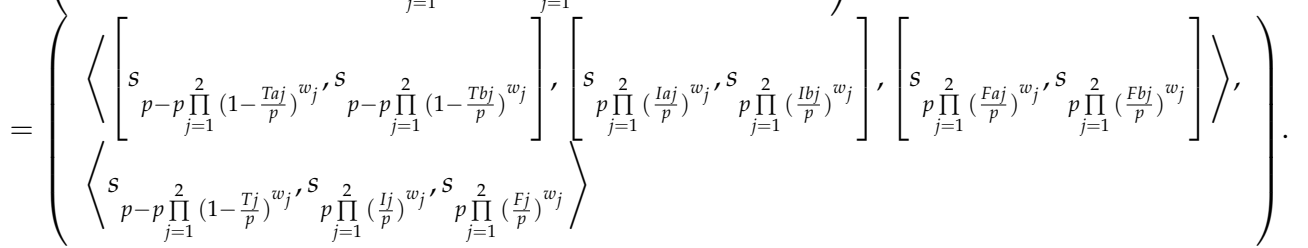

(2) Set $n=k$, by Equation (5) we obtain

$$
\begin{aligned}
& \operatorname{LNCNWAA}\left(h_{1}, h_{2}, \cdots, h_{k}\right)=\sum_{j=1}^{k} w_{j} h_{j}
\end{aligned}
$$

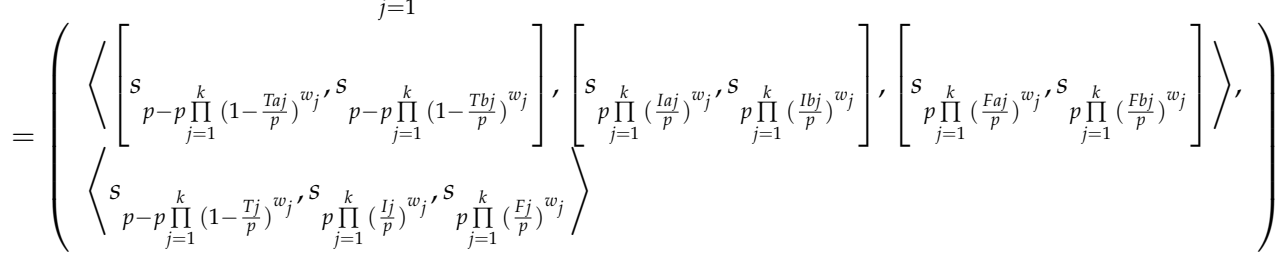


(3) Set $n=k+1$, based on Equations (6) and (7), we can obtain the following result:

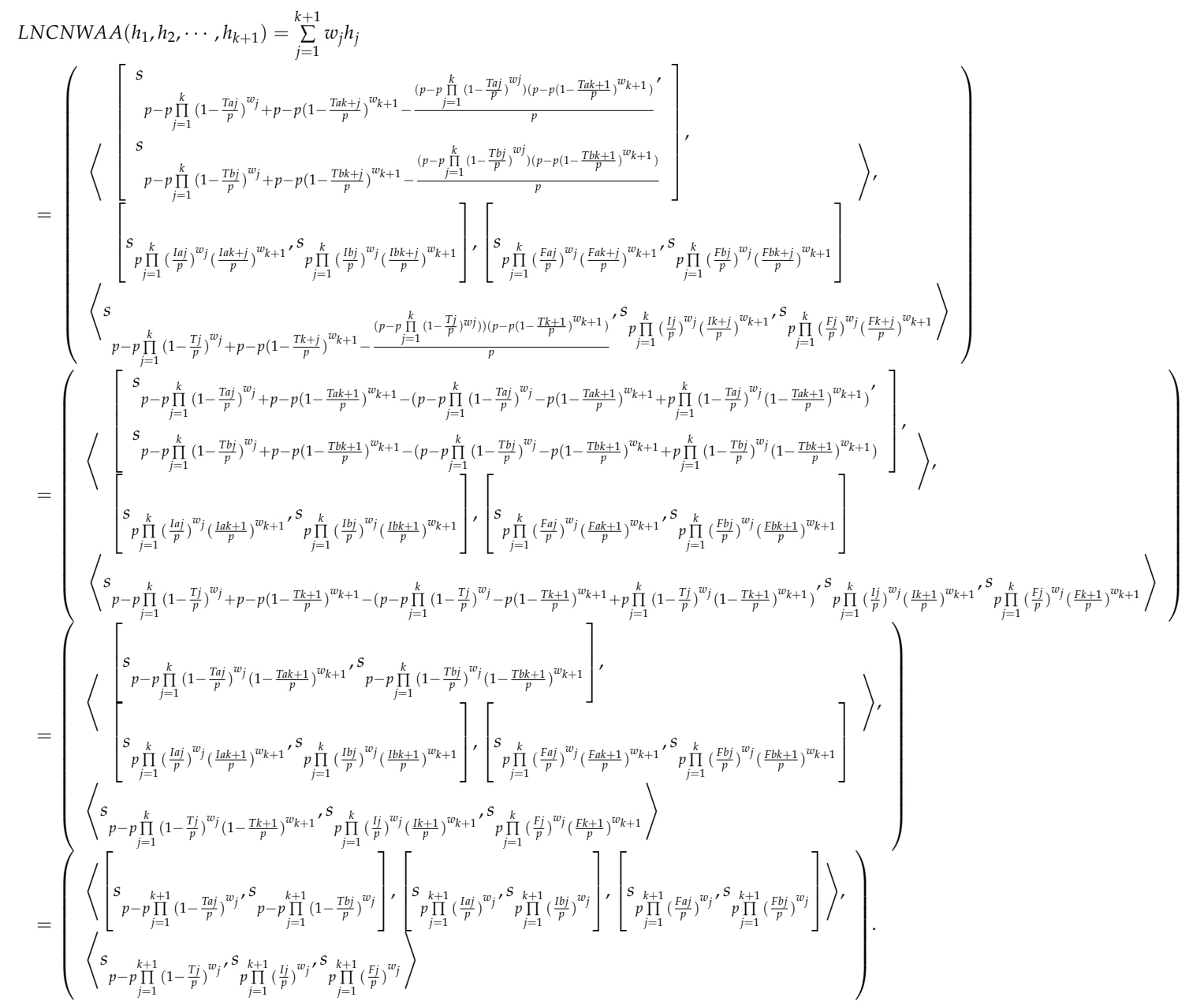

Based on the above results, Equation (5) can hold for any $n$. The proof is finished.

Clearly, the LNCNWAA operator contains the following properties:

(1) Idempotency: Let $h_{j}(j=1,2, \ldots, n)$ be a group of LNCNs. When $h_{j}=h$ for $j=1,2, \ldots, n$, there is $\operatorname{LNCNWAA}\left(h_{1}, h_{2}, \cdots, h_{n}\right)=h$.

(2) Boundedness: Let $h_{j}(j=1,2, \ldots, n)$ be a group of LNCNs and the minimum and maximum LNCNs be $h^{-}=\left(\left\langle\left[\min _{j}\left(s_{T a j}\right), \min _{j}\left(s_{T b j}\right)\right],\left[\max _{j}\left(s_{I a j}\right), \max _{j}\left(s_{I b j}\right)\right],\left[\max _{j}\left(s_{F a j}\right), \max _{j}\left(s_{F b j}\right)\right]\right\rangle,\left\langle\min _{j}\left(s_{T j}\right), \max _{j}\left(s_{I j}\right), \max _{j}\left(s_{F j}\right)\right\rangle\right)$ and $h^{+}=\left(\left\langle\left[\max _{j}\left(s_{T a j}\right), \max _{j}\left(s_{T b j}\right)\right],\left[\min _{j}\left(s_{I a j}\right), \min _{j}\left(s_{I b j}\right)\right],\left[\min _{j}\left(s_{F a j}\right), \min _{j}\left(s_{F b j}\right)\right]\right\rangle,\left\langle\max _{j}\left(s_{T j}\right), \min _{j}\left(s_{I j}\right), \min _{j}\left(s_{F j}\right)\right\rangle\right)$ respectively. Then, there exists $h^{-} \leq \operatorname{LNCNWAA}\left(h_{1}, h_{2}, \cdots, h_{n}\right) \leq h^{+}$.

(3) Monotonicity: Let $h_{j}(j=1,2, \ldots, n)$ be a group of LNCNs. When $h_{j} \leq h_{j}^{*}$ for $j=1,2, \ldots, n$, then there exists $\operatorname{LNCNWAA}\left(h_{1}, h_{2}, \cdots, h_{n}\right) \leq \operatorname{LNCNWAA}\left(h_{1}^{*}, h_{2}^{*}, \cdots, h_{n}^{*}\right)$. 
Proof. (1) For $h_{j}=h(j=1,2, \ldots, n)$, we have the following result:

$$
\begin{aligned}
& \operatorname{LNCNWAA}\left(h_{1}, h_{2}, \cdots, h_{n}\right)=\sum_{j=1}^{n} w_{j} h_{j}
\end{aligned}
$$

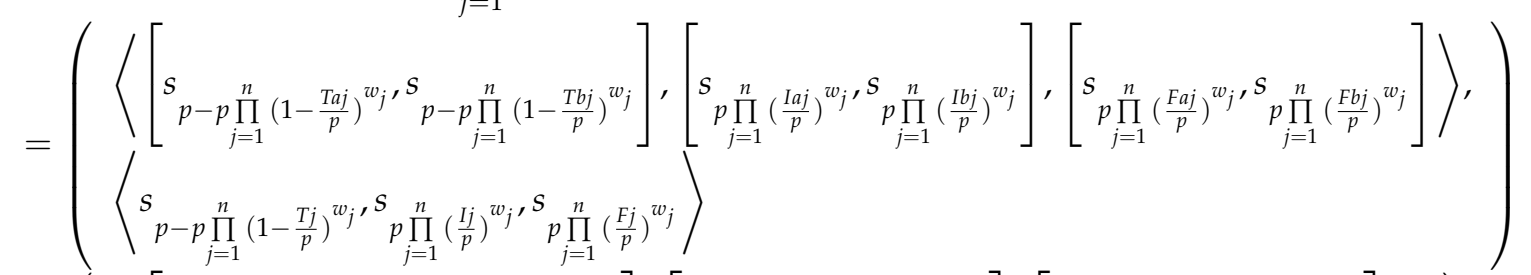

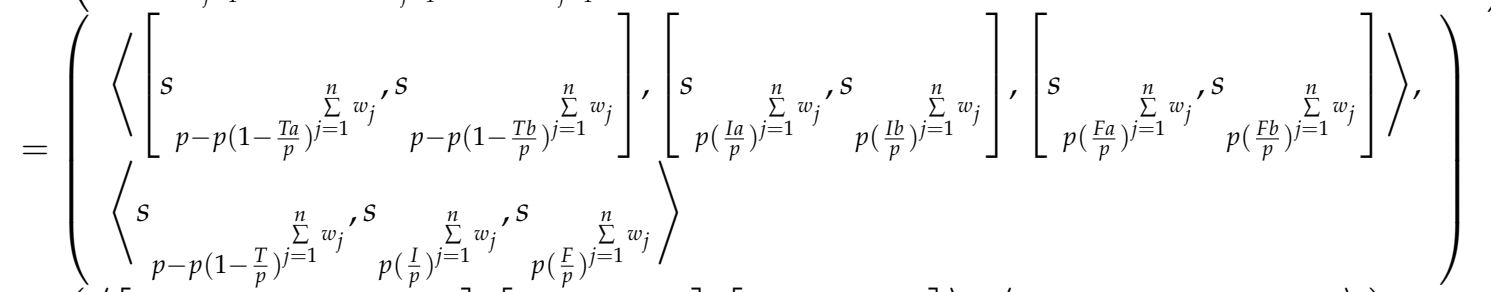

$$
\begin{aligned}
& =\left(\left\langle\left[s_{p-p\left(1-\frac{T a}{p}\right)}, s_{p-p\left(1-\frac{T b}{p}\right)}\right],\left[s_{p\left(\frac{I a}{p}\right)}, s_{p\left(\frac{I b}{p}\right)}\right],\left[s_{p\left(\frac{F a}{p}\right)}, s_{p\left(\frac{F b}{p}\right)}\right]\right\rangle,\left\langle s_{p-p\left(1-\frac{T}{p}\right)}, s_{p\left(\frac{I}{p}\right)}, s_{p\left(\frac{F}{p}\right)}\right\rangle\right) \\
& =\left(\left\langle\left[s_{T a}, s_{T b}\right],\left[s_{I a}, s_{I b}\right],\left[s_{F a}, s_{F b}\right]\right\rangle,\left\langle s_{T}, s_{I}, s_{F}\right\rangle\right)=h \text {. }
\end{aligned}
$$

(2) Since the minimum LNCN is $h^{-}$and the maximum LNCN is $h^{+}$, there is $h^{-} \leq h_{j} \leq h^{+}$. Thus, there exists $\sum_{j=1}^{n} w_{j} h^{-} \leq \sum_{j=1}^{n} w_{j} h_{j} \leq \sum_{j=1}^{n} w_{j} h^{+}$. According to the above property (1), there exists $h^{-} \leq \sum_{j=1}^{n} w_{j} h_{j} \leq h^{+}$. Then, $h^{-} \leq \operatorname{LNCNWAA}\left(h_{1}, h_{2}, \cdots, h_{n}\right) \leq h^{+}$can hold

(3) For $h_{j} \leq h_{j}^{*}(j=1,2, \ldots, n)$, there exists $\sum_{j=1}^{n} w_{j} h_{j} \leq \sum_{j=1}^{n} w_{j} h_{j}^{*}$. Then, $\operatorname{LNCNWAA}\left(h_{1}, h_{2}, \cdots, h_{n}\right) \leq \operatorname{LNCNWAA}\left(h_{1}^{*}, h_{2}^{*}, \cdots, h_{n}^{*}\right)$ can hold.

Hence, we complete the proofs of these properties.

Obviously, when $w_{j}=1 / n$ for $j=1,2, \ldots, n$, the LNCNWAA operator is reduced to the LNCN arithmetic averaging operator.

\subsection{LNCNWGA Operator}

Definition 7. Let $h_{j}=\left(\left\langle\left[s_{T a j}, s_{T b j}\right],\left[s_{I a j}, s_{I b j}\right],\left[s_{F a j}, s_{F b j}\right]\right\rangle,\left\langle s_{T j}, s_{I j}, s_{F j}\right\rangle\right)(j=1,2, \ldots, n)$ be a group of LNCNs, then the LNCNWGA operator is defined as follows:

$$
\operatorname{LNCNWGA}\left(h_{1}, h_{2}, \cdots, h_{n}\right)=\prod_{j=1}^{n} h_{j}^{w_{j}}
$$

where $w_{j}$ is the weight of $h_{j}(j=1,2, \ldots, n)$ for $w_{j} \in[0,1]$ and $\sum_{j=1}^{n} w_{j}=1$.

According to Definitions 3 and 7, we can introduce the following theorem. 
Theorem 2. Leth $_{j}=\left(\left\langle\left[s_{T a j}, s_{T b j}\right],\left[s_{I a j}, s_{I b j}\right],\left[s_{F a j}, s_{F b j}\right]\right\rangle,\left\langle s_{T j}, s_{I j}, s_{F j}\right\rangle\right)(j=1,2, \ldots, n)$ be a group of LNCNs. Then, the aggregation result of Equation (8) is still a LNCN, which is calculated by the following aggregation equation:

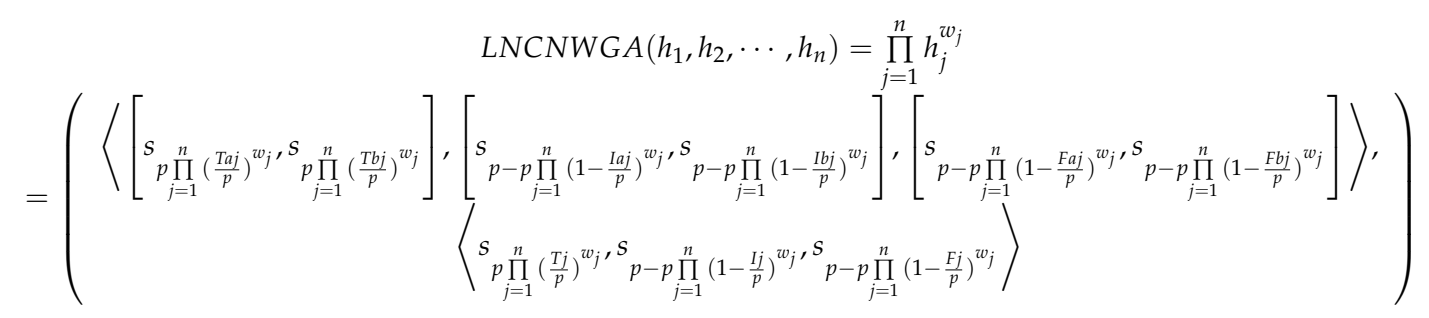

where $w_{j}$ is the weight of $h_{j}(j=1,2, \ldots, n)$ for $w_{j} \in[0,1]$ and $\sum_{j=1}^{n} w_{j}=1$. Obviously, when $w_{j}=1 / n$ for $j=1,2, \ldots, n$, the LNCNWGA operator is reduced to the LNCN geometric averaging operator.

Based on the similar proof manner of Theorem 1, we can prove Theorem 2. Hence, it is omitted here.

Obviously, the LNCNWGA operator also contains the following properties:

(1) Idempotency: Let $h_{j}(j=1,2, \ldots, n)$ be a group of LNCNs. When $h_{j}=h$ for $j=1,2, \ldots, n$, there exists $\operatorname{LNCNWGA}\left(h_{1}, h_{2}, \cdots, h_{n}\right)=h$.

(2) Boundedness: Let $h_{j}(j=1,2, \ldots, n)$ be a group of LNCNs and the minimum and maximum LNCNs be $h^{-}=\left(\left\langle\left[\min _{j}\left(s_{T a j}\right), \min _{j}\left(s_{T b j}\right)\right],\left[\max _{j}\left(s_{I a j}\right), \max _{j}\left(s_{I b j}\right)\right],\left[\max _{j}\left(s_{F a j}\right), \max _{j}\left(s_{F b j}\right)\right]\right\rangle,\left\langle\min _{j}\left(s_{T j}\right), \max _{j}\left(s_{I j}\right), \max _{j}\left(s_{F j}\right)\right\rangle\right)$ and $h^{+}=\left(\left\langle\left[\max _{j}\left(s_{T a j}\right), \max _{j}\left(s_{T b j}\right)\right],\left[\min _{j}\left(s_{I a j}\right), \min _{j}\left(s_{I b j}\right)\right],\left[\min _{j}\left(s_{F a j}\right), \min _{j}\left(s_{F b j}\right)\right]\right\rangle,\left\langle\max _{j}\left(s_{T j}\right), \min _{j}\left(s_{I j}\right), \min _{j}\left(s_{F j}\right)\right\rangle\right)$ respectively. Then, there exists $h^{-} \leq \operatorname{LNCNWGA}\left(h_{1}, h_{2}, \cdots, h_{n}\right) \leq h^{+}$.

(3) Monotonicity: Let $h_{j}(j=1,2, \ldots, n)$ be a group of LNCNs. When $h_{j} \leq h_{j}^{*}$ for $j=1,2, \ldots, n$, there exists $\operatorname{LNCNWGA}\left(h_{1}, h_{2}, \cdots, h_{n}\right) \leq \operatorname{LNCNWGA}\left(h_{1}^{*}, h_{2}^{*}, \cdots, h_{n}^{*}\right)$.

Based on the similar proofs of the properties corresponding to the LNCNWAA operator, we can also prove these properties of the LNCNWGA operator. Hence, these proofs are omitted here.

\section{Decision-Making Method Based on the LNCNWAA or Linguistic Neutrosophic Cubic Number Weighted Geometric Averaging (LNCNWGA) Operator}

This section proposes a decision-making method based the LNCNWAA or LNCNWGA operator to solve multiple attribute decision-making problems with LNCN information.

If there is a multiple attribute decision-making problem, we consider $Q=\left\{Q_{1}, Q_{2}, \ldots, Q_{m}\right\}$ as a set of alternatives and $R=\left\{R_{1}, R_{2}, \ldots, R_{n}\right\}$ as a set of attributes. The weigh vector of the attributes $R_{j}(j=1,2, \ldots, n)$ is specified as $w=\left(w_{1}, w_{2}, \ldots, w_{n}\right)$. Then, decision-makers are invited to evaluate the alternatives $Q_{i}(i=1,2, \ldots, m)$ over the attributes $R_{j}(j=1,2, \ldots, n)$ by LNCNs from the predefined linguistic term set $S=\left\{s_{j} \mid j \in[0, p]\right\}$, where $p+1$ is an odd number/cardinality. Based on the linguistic term set, the decision-makers can assign the uncertain linguistic arguments corresponding to the truth, indeterminacy, and falsity linguistic terms and the certain linguistic arguments corresponding to the truth, indeterminacy, and falsity linguistic terms in each LNCN as the linguistic evaluation of each attribute $R_{j}(j=1,2, \ldots, n)$ on each alternative $Q_{i}(i=1,2, \ldots, m)$ in the evaluation process. Thus, all the LNCNs can be constructed as a LNCN decision matrix $D=\left(h_{i j}\right)_{m \times n}$, where $h_{i j}=\left(\left\langle\left[s_{T a i j}, s_{T b i j}\right],\left[s_{I a i j}, s_{I b i j}\right],\left[s_{F a i j}, s_{F b i j}\right]\right\rangle,\left\langle s_{T i j}, s_{I i j}, s_{F i j}\right\rangle\right)(i=1,2, \ldots, m ; j=1,2, \ldots, n)$ is a LNCN.

Thus, the decision-making method based on the LNCNWAA or LNCNWGA operator is described by the following decision steps:

Step 1 Calculate $h_{i}=\operatorname{LNCNWAA}\left(h_{i 1}, h_{i 2}, \ldots, h_{i n}\right)$ or $h_{i}=\operatorname{LNCNWGA}\left(h_{i 1}, h_{i 2}, \ldots, h_{i n}\right)(i=1,2, \ldots, m)$ by using Equation (5) or Equation (9) and obtain the collective overall LNCN $h_{i}$ for $Q_{i}(i=1,2$, $\ldots, m)$. 
Step 2 Calculate the values of $S\left(h_{i}\right)\left(H\left(h_{i}\right)\right.$ and / or $C\left(h_{i}\right)$ if necessary) $(i=1,2, \ldots, m)$ for each collective overall LNCN $h_{i}(i=1,2, \ldots, m)$ by Equation (1) (Equation (2) and/or Equation (3)).

Step 3 Rank the alternatives corresponding to the ranking method of Definition 5, and then select the best one.

Step 4 End.

\section{Illustrative Example}

This section provides an illustrative example in order to demonstrate the application of the proposed decision-making method under a linguistic neutrosophic cubic environment.

A manufacturing company needs to hire a mechanical designer. After all applicants are chosen preliminarily by the human resources department, four potential candidates $Q_{1}, Q_{2}, Q_{3}$, and $Q_{4}$ need to be further evaluated according to the three requirements/attributes: (1) $R_{1}$ is the innovation skill; (2) $R_{2}$ is the design experience; (3) $R_{3}$ is the self-confidence. A group of experts is required to conduct the interview and to choose the most suitable candidate. Then, the weigh vector $w=(0.45,0.35,0.2)$ is considered as the importance of the three attributes. Herewith, the experts (decision-makers) need to evaluate the four potential candidates/alternatives $Q_{i}(i=1,2,3,4)$ corresponding to the three attributes $R_{j}(j=1,2,3)$ by the form of LNCNs based on the given linguistic term set $S=\left\{s_{j} \mid j \in[0, p]\right\}$, where $S=\left\{s_{0}=\right.$ extremely poor, $s_{1}=$ very poor, $s_{2}=$ poor, $s_{3}=$ slightly poor, $s_{4}=$ fair, $s_{5}=$ slightly good, $s_{6}=$ good, $s_{7}=$ very good, $s_{8}=$ extremely good $\}$ for $p=8$. Thus, all the LNCNs are given by the experts and constructed as the following LNCN decision matrix $D\left(h_{i j}\right)_{4 \times 3}$ :

$$
D\left(h_{i j}\right)_{4 \times 3}=\left[\begin{array}{llll}
\left(\left\langle\left[s_{4}, s_{6}\right],\left[s_{1}, s_{2}\right],\left[s_{1}, s_{3}\right]\right\rangle,\left\langle s_{5}, s_{1}, s_{2}\right\rangle\right) & \left(\left\langle\left[s_{4}, s_{6}\right],\left[s_{1}, s_{3}\right],\left[s_{1}, s_{3}\right]\right\rangle,\left\langle s_{5}, s_{2}, s_{2}\right\rangle\right) & \left(\left\langle\left[s_{4}, s_{7}\right],\left[s_{1}, s_{3}\right],\left[s_{2}, s_{3}\right]\right\rangle,\left\langle s_{6}, s_{2}, s_{3}\right\rangle\right) \\
\left(\left\langle\left[s_{3}, s_{5}\right],,\left[s_{1}, s_{2}\right],\left[s_{1}, s_{2}\right]\right\rangle,\left\langle s_{4}, s_{1}, s_{1}\right\rangle\right) & \left(\left\langle\left[s_{5}, s_{7}\right],,\left[s_{1}, s_{2}\right],\left[s_{1}, s_{2}\right]\right\rangle,\left\langle s_{6}, s_{1}, s_{2}\right\rangle\right) & \left(\left\langle\left[s_{4}, s_{6}\right],\left[s_{2}, s_{3}\right],\left[s_{1}, s_{2}\right]\right\rangle,\left\langle s_{5}, s_{3}, s_{1}\right\rangle\right) \\
\left(\left\langle\left[s_{4}, s_{7}\right],,\left[s_{1}, s_{2}\right],\left[s_{2}, s_{3}\right]\right\rangle,\left\langle s_{5}, s_{2}, s_{3}\right\rangle\right) & \left(\left\langle\left[s_{6}, s_{7}\right],\left[s_{1}, s_{3}\right],\left[s_{1}, s_{3}\right]\right\rangle,\left\langle s_{7}, s_{2}, s_{2}\right\rangle\right) & \left(\left\langle\left[s_{5}, s_{7}\right],\left[s_{1}, s_{3}\right],\left[s_{2}, s_{3}\right]\right\rangle,\left\langle s_{5}, s_{2}, s_{3}\right\rangle\right) \\
\left(\left\langle\left[s_{6}, s_{7}\right],\left[s_{2}, s_{3}\right],\left[s_{2}, s_{3}\right]\right\rangle,\left\langle s_{7}, s_{3}, s_{3}\right\rangle\right) & \left(\left\langle\left[s_{5}, s_{7}\right],\left[s_{1}, s_{2}\right],\left[s_{1}, s_{2}\right]\right\rangle,\left\langle s_{6}, s_{1}, s_{2}\right\rangle\right) & \left(\left\langle\left[s_{4}, s_{6}\right],\left[s_{1}, s_{2}\right],\left[s_{1}, s_{2}\right]\right\rangle,\left\langle s_{5}, s_{1}, s_{1}\right\rangle\right)
\end{array}\right]
$$

Thus, the proposed decision-making method can be applied to the decision-making problem with LNCN information.

On the one hand, we can use the decision-making method based on the LNCNWAA operator, which is described by the following decision steps:

Step 1 By using Equation (5), the collective overall LNCNs of $h_{i}$ for $Q_{i}(i=1,2,3,4)$ can be given as follows:

$h_{1}=\left(\left\langle\left[s_{4}, s_{6.2589}\right],\left[s_{1}, s_{2.4997}\right],\left[s_{1.1487}, s_{3}\right]\right\rangle,\left\langle s_{5.2337}, s_{1.4641}, s_{2.1689}\right\rangle\right), h_{2}=\left(\left[s_{4.0011}, s_{6.1167}\right],\left[s_{1.1487}\right.\right.$, $\left.s_{2.1689}\right],\left[s_{1}, s_{2}\right]>,\left\langle s_{5.0371}, s_{1.2457}, s_{1.2746}>\right), h_{3}=\left(\left[s_{5.0371}, s_{7}\right],\left[s_{1}, s_{2.4997}\right],\left[s_{1.5692}, s_{3}\right]\right\rangle,<s_{5.9577}, s_{2}$, $\left.s_{2.6031}>\right)$, and $h_{4}=\left(\left[s_{5.3523}, s_{6.8513}\right],\left[s_{1.366}, s_{2.4003}\right],\left[s_{1.366}, s_{2.4003}\right]>,<s_{6.4122}, s_{1.6395}, s_{2.0896}>\right)$.

Step 2 Calculate the score values of $S\left(h_{i}\right)(i=1,2,3,4)$ by Equation (1):

$$
S\left(h_{1}\right)=0.7252, S\left(h_{2}\right)=0.7544, S\left(h_{3}\right)=0.7406 \text {, and } S\left(h_{4}\right)=0.7688 \text {. }
$$

Step 3 The ranking order of the four alternatives is $Q_{4} \succ Q_{2} \succ Q_{3} \succ Q_{1}$ based on the score values. Thus, the candidate $Q_{4}$ is the best choice among the four candidates.

On the other hand, we can also use the decision-making method based on the LNCNWGA operator, which is described by the following decision procedures:

Step 1' By using Equation (9), the collective overall LNCNs of $h_{i}$ for $Q_{i}(i=1,2,3,4)$ are given as follows:

$\left.\left.h_{1}=\left(<\left[s_{4}, s_{6.1879}\right],\left[s_{1}, s_{2.5725}\right],\left[s_{1.2125}, s_{3}\right]\right\rangle,<s_{5.1857}, s_{1.569}, s_{2.2148}\right\rangle\right), h_{2}=\left(<\left[s_{3.7998}, s_{5.8338}\right],\left[s_{1.2125}\right.\right.$, $\left.\left.\left.\left.s_{2.2148}\right],\left[s_{1}, s_{2}\right]\right\rangle,<s_{4.8203}, s_{1.4556}, s_{1.3677}\right\rangle\right), h_{3}=\left(<\left[s_{4.8203}, s_{7}\right],\left[s_{1}, s_{2.5725}\right],\left[s_{1.6674}, s_{3}\right]\right\rangle,<s_{5.6249}, s_{2}$, $\left.\left.s_{2.6705}\right\rangle\right)$, and $\left.\left.h_{4}=\left(<\left[s_{5.1906}, s_{6.7875}\right],\left[s_{1.4691}, s_{2.4726}\right],\left[s_{1.4691}, s_{2.4726}\right]\right\rangle,<s_{6.2007}, s_{1.9835}, s_{2.2996}\right\rangle\right)$.

Step 2' By using Equation (1), we calculate the score values of $S\left(h_{i}\right)(i=1,2,3,4)$ as follows:

$$
S\left(h_{1}\right)=0.7195, S\left(h_{2}\right)=0.7389, S\left(h_{3}\right)=0.7296 \text {, and } S\left(h_{4}\right)=0.7502 \text {. }
$$


Step 3' The ranking order of the four candidates is $Q_{4} \succ Q_{2} \succ Q_{3} \succ Q_{1}$. Thus, the candidate $Q_{4}$ is still the best choice among the four candidates.

Obviously, the above two ranking orders based on the LNCNWAA and LNCNWGA operators and the best candidate are identical.

Compared with existing currant linguistic neutrosophic decision-making methods [16,17], the decision information in this study is LNCNs, while the decision information used in $[16,17]$ is linguistic neutrosophic numbers. As mentioned above, since LNCN is composed of its uncertain neutrosophic number and its linguistic neutrosophic number, $\mathrm{LNCN}$ contains more information than the unique linguistic neutrosophic number in $[16,17]$. However, existing linguistic neutrosophic decision-making methods in $[16,17]$ cannot handle such a decision-making problem with linguistic neutrosophic cubic information in this paper. Therefore, the decision-making method proposed in this paper can solve decision-making problems with both certain linguistic and uncertain linguistic neutrosophic information. It can also provide a new way for hybrid linguistic decision-making problems under certain and uncertain linguistic environments.

Due to no related studies in the existing literature, this is the first study to propose a new concept of LNCN and a new linguistic neutrosophic cubic decision-making method. However, decision-makers can select one of two weighted aggregation operators of LNCNs to solve linguistic neutrosophic cubic decision-making problems according to their preference and actual requirements.

\section{Conclusions}

This paper originally proposed the concept of LNCN, including the internal LNCN and external LNCN, and the operational laws of LNCNs, and introduced the score, accuracy, and certain functions of LNCNs for comparing/ranking LNCNs. Then, we proposed the LNCNWAA and LNCNWGA operators to aggregate LNCNs and discussed their properties. Next, we developed a multiple attribute decision-making method based on the LNCNWAA or LNCNWGA operator for solving multiple attribute decision-making problems with LNCN information. Finally, an example illustrated the application of the developed method under a LNCN environment. The proposed decision-making method can solve decision-making problems with determinate and uncertain linguistic neutrosophic arguments.

Obviously, the main advantages of this study are summarized as follows:

(1) The LNCN expression is superior to existing linguistic expressions in the certain and uncertain linguistic environment.

(2) The developed linguistic neutrosophic cubic decision-making method extends existing ones to deal with linguistic neutrosophic cubic decision-making problems with the hybrid information of both uncertain linguistic neutrosophic arguments and certain linguistic neutrosophic arguments.

(3) The developed new method enriches linguistic neutrosophic expressions and linguistic neutrosophic decision-making methods.

In the future work, we shall further introduce new aggregation operators of LNCNs and applications in group decision-making, pattern recognition, and medical diagnoses.

Acknowledgments: This paper was supported by the National Natural Science Foundation of China (Nos. 71471172, 61703280).

Conflicts of Interest: The author declares no conflict of interest.

\section{References}

1. Zadeh, L.A. The concept of a linguistic variable and its application to approximate reasoning Part I. Inf. Sci. 1975, 8, 199-249. [CrossRef]

2. Herrera, F.; Herrera-Viedma, E.; Verdegay, L. A model of consensus in group decision making under linguistic assessments. Fuzzy Sets Syst. 1996, 79, 73-87. [CrossRef] 
3. Herrera, F.; Herrera-Viedma, E. Linguistic decision analysis: Steps for solving decision problems under linguistic information. Fuzzy Sets Syst. 2000, 115, 67-82. [CrossRef]

4. $\mathrm{Xu}, \mathrm{Z}$.S. A method based on linguistic aggregation operators for group decision making with linguistic preference relations. Inf. Sci. 2004, 166, 19-30. [CrossRef]

5. Xu, Z.S. A note on linguistic hybrid arithmetic averaging operator in multiple attribute group decision making with linguistic information. Group Decis. Negot. 2006, 15, 593-604. [CrossRef]

6. Xu, Z.S. Uncertain linguistic aggregation operators based approach to multiple attribute group decision making under uncertain linguistic environment. Inf. Sci. 2004, 168, 171-184. [CrossRef]

7. Xu, Z.S. Induced uncertain linguistic OWA operators applied to group decision making. Inf. Fusion 2006, 7, 231-238. [CrossRef]

8. Wei, G.W. Uncertain linguistic hybrid geometric mean operator and its application to group decision making under uncertain linguistic environment. Int. J. Uncertain. Fuzziness Knowl. Based Syst. 2009, 17, 251-267. [CrossRef]

9. Park, J.H.; Gwak, M.G.; Kwun, Y.C. Uncertain linguistic harmonic mean operators and their applications to multiple attribute group decision making. Computing 2011, 93, 47. [CrossRef]

10. Wei, G.W.; Zhao, X.F.; Lin, R.; Wang, H.J. Uncertain linguistic Bonferroni mean operators and their application to multiple attribute decision making. Appl. Math. Modell. 2013, 37, 5277-5285. [CrossRef]

11. Zhang, H. Uncertain linguistic power geometric operators and their use in multiattribute group decision making. Math. Probl. Eng. 2015, 2015, 948380. [CrossRef]

12. Zhang, H. Linguistic intuitionistic fuzzy sets and application in MAGDM. J. Appl. Math. 2014, $2014,432092$. [CrossRef]

13. Chen, Z.C.; Liu, P.H.; Pei, Z. An approach to multiple attribute group decision making based on linguistic intuitionistic fuzzy numbers. Int. J. Comput. Intell. Syst. 2015, 8, 747-760. [CrossRef]

14. Peng, H.G.; Wang, J.Q.; Cheng, P.F. A linguistic intuitionistic multi-criteria decision-making method based on the Frank Heronian mean operator and its application in evaluating coal mine safety. Int. J. Mach. Learn. Cybern. 2017. [CrossRef]

15. Ye, J. Aggregation operators of neutrosophic linguistic numbers for multiple attribute group decision making. SpringerPlus 2016, 5, 1691. [CrossRef] [PubMed]

16. Fang, Z.B.; Ye, J. Multiple attribute group decision-making method based on linguistic neutrosophic numbers. Symmetry 2017, 9, 111. [CrossRef]

17. Li, Y.Y.; Zhang, H.Y.; Wang, J.Q. Linguistic neutrosophic sets and their application in multicriteria decision-making problems. Int. J. Uncertain. Quantif. 2017, 7, 135-154. [CrossRef]

18. Jun, Y.B.; Kim, C.S.; Yang, K.O. Cubic sets. Ann. Fuzzy Math. Inform. 2012, 4, 83-98.

19. Ali, M.; Deli, I.; Smarandache, F. The theory of neutrosophic cubic sets and their applications in pattern recognition. J. Intell. Fuzzy Syst. 2016, 30, 1957-1963. [CrossRef]

20. Jun, Y.B.; Smarandache, F.; Kim, C.S. Neutrosophic cubic sets. New Math. Nat. Comput. 2017, 13, 41-45. [CrossRef]

21. Banerjee, D.; Giri, B.C.; Pramanik, S.; Smarandache, F. GRA for multi attribute decision making in neutrosophic cubic set environment. Neutrosoph. Sets Syst. 2017, 15, 64-73.

22. Lu, Z.K.; Ye, J. Cosine measures of neutrosophic cubic sets for multiple attribute decision-making. Symmetry 2017, 9, 121. [CrossRef]

(C) 2017 by the author. Licensee MDPI, Basel, Switzerland. This article is an open access article distributed under the terms and conditions of the Creative Commons Attribution (CC BY) license (http:/ / creativecommons.org/licenses/by/4.0/). 